\title{
Effect of folate treatment on homocysteinemia in cardiac patients: A prospective study
}

\author{
K. N. Mehta, M. C. Chag*, K. H. Parikh*, U. G. Shah, H. A. Baxi, A. H. Chandarana*, A. M. Naik*, \\ R. K. Goyal
}

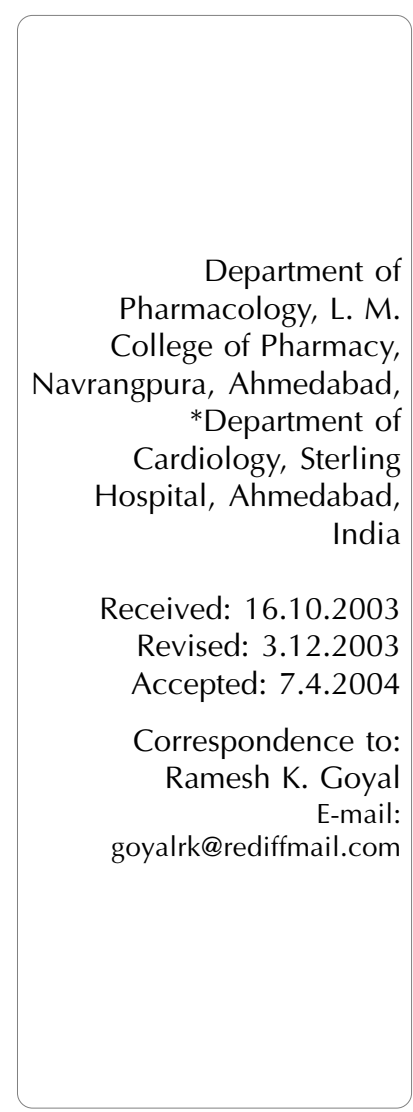

\begin{abstract}
Objective: To study the effect of folate treatment on homocysteine (HCY) levels in patients with Coronary Artery Disease (CAD). We also made an attempt to analyze HCY levels in relation to other risk factors present in these patients.

Materials and Methods: The study was a randomised, non-blind clinical trial carried out in 84 angiographically documented CAD patients and 40 controls (angiographically proved normal or treadmill test negative). Biochemical analyses of glucose, insulin, HCY, lipid profile and C-Reactive Proteins (CRP) were conducted in serum samples collected from these patients. Treatment with folic acid ( $5 \mathrm{mg} /$ day) for 3 months was given to patients with hyperhomocysteinemia and the posttreatment HCY levels were analyzed.

Results: The mean plasma HCY level in control volunteers was found to be $17.85 \pm 1.4 \mu \mathrm{mol} / \mathrm{L}$, which was significantly lower than those in CAD patients $(24.69 \pm 2.8 \mu \mathrm{mol} / \mathrm{L})$. The levels were higher in non-diabetic patients $(30.33 \pm 3.9 \mu \mathrm{mol} / \mathrm{L})$ as compared to non-diabetic controls $(18.18 \pm 1.6$ $\mu \mathrm{mol} / \mathrm{L})$ as well as diabetic patients $(14.53 \pm 1.43 \mu \mathrm{mol} / \mathrm{L})$, irrespective of the presence of hypertension. No correlation was found between total HCY (t-HCY) levels and serum glucose, insulin, triglycerides, VLDL, LDL, HDL, cholesterol or CRP levels. A negative correlation was found between $\mathrm{t}-\mathrm{HCY}$ levels and the age of the patients. The HCY levels were highest in the younger age group of patients (30-40 years) $(49 \pm 7.8 \mu \mathrm{mol} / \mathrm{L})$. There was a significant reduction in t-HCY levels after the folate treatment in mild and moderate hyperhomocystienemic patients but the reduction in patients with severe hyperhomocysteinemia was not significant.

Conclusion: CAD patients have higher HCY levels than subjects without CAD. Indian diabetic patients have lower levels of t-HCY irrespective of the presence of cardiac disease. Folate treatment decreases the t-HCY levels in homocysteinemia and thus can be used in treatment of homocysteinemia.
\end{abstract}

KEY WORDS: Coronary artery disease, diabetes mellitus, folic acid

\section{Introduction}

Coronary Artery Disease (CAD) is one of the most important causes of premature death in industrialized countries. ${ }^{[1]}$ It is estimated that CAD will emerge as the single largest contributor to mortality in India, accounting for nearly one-third of all deaths. ${ }^{[2]}$ The emigrant South Asian has myocardial infarction 2.5 to 5 times more frequently and 1.5 to 3 times higher from total CAD compared to the endemic population of Singapore, South Africa, England and USA. ${ }^{[1]}$ Moreover, CAD among Indians has been found to be severe, more diffuse, associated with serious complications and increasing mortality at a younger age. ${ }^{[3]}$

Conventional risk factors studied in the western population do not explain the incidence of CAD in Indians. The four major risk factors for CAD viz. hyperlipidemia, hypertension, diabetes mellitus and cigarette smoking, which were present in Framingham cohort are often not very prominent among Indians with CAD. Instead, CAD is present in Indians with relatively lower cholesterol and triglyceride levels. ${ }^{[4]}$ In contrast, insulin resistance seems to be much more prevalent in Indians. ${ }^{[5]}$ Hyperinsulinemia, impaired glucose tolerance, 
hypertriglyceridemia, abdominal obesity (apple body configuration) and hypertension, the combination commonly referred to as "Metabolic Syndrome X", is important in the Indian context. In addition, high lipoprotein- (a) [Lp (a)] and atherogenic phenotype are important in Indians. ${ }^{[6]}$ Evidence is emerging in favor of newer risk factors like high plasma levels of tissue plasminogen activator inhibitor (t PAI-1), ${ }^{[7]}$ low levels of apo A-1, high levels of apo B, intermediate density lipoprotein and LDL subclass pattern B. ${ }^{[8]}$

Recently it has been established that hyperhomocysteinemia is also one of the risk factors of CAD. To date, observations in 100 epidemiological clinical studies have suggested that elevated blood HCY level is a risk factor for atherosclerotic vascular disease (AVD). ${ }^{[9,10]}$ Boushey et al ${ }^{[11]}$ found that an increment of $5 \mu \mathrm{mol} / \mathrm{L}$ in plasma HCY concentration increases the incidence of CAD by $60 \%$ to $80 \%$. It has been reported that each three-unit increase in HCY equals a 35\% increase in myocardial infarction. The possible mechanism of hyperhomocysteinemia related vascular injury is still a subject of much research. After the description of the characteristic atherosclerotic lesions in children with homocystinuria by McCully, ${ }^{[13]}$ numerous studies have explored sites of adverse influence of HCY, including the endothelial surface, vascular smooth muscle cells, connective tissue, interactions with plasma lipoproteins, clotting factors and platelets. HCY causes the smooth muscle cells that support the arterial wall, to multiply a part of the atherogenic process. ${ }^{[14]}$ In its reactive form, homocysteine thiolactone, HCY causes platelets to aggregatewhich is a part of the clotting process. HCY changes coagulation factor levels so as to encourage blood clot formation. It also prevents small arteries from dilating, thereby making them more vulnerable to obstruction by clot or plaque. ${ }^{[15]}$

In general, case-control studies have been robust in confirming the association between hyperhomocysteinemia and CAD. ${ }^{[10,16]}$ However, there exists controversy with respect to hyperhomocysteinemia and CAD. There could be a possibility of ethnic differences as well. A population-based study of South Indians in Canada ${ }^{[17]}$ measured HCY in 342 participants and found mean fasting levels to be $11.22 \mu \mathrm{mol} / \mathrm{L}$. This was significantly higher than European and Chinese participants in the same study. Recent studies have shown that plasma HCY concentrations are higher in Asian migrants in Europe as compared to native European whites ${ }^{[18]}$ and also confirm that HCY is a risk factor for CAD in this racial group. ${ }^{[18]}$ A study carried out in South Indian population concluded that elevated HCY levels are not associated with CAD in South Indian male subjects with or without diabetes ${ }^{[19]}$ whereas a prospective study from North India concluded that plasma HCY is a significant independent risk factor for angiographic CAD, irrespective of the baseline lipid profile of the patient. ${ }^{\mid 20]}$

Other factors such as age, gender, smoking, diabetes mellitus, dyslipidemia etc. can also alter the relationship between hyperhomocysteinemia and CAD. Diabetes mellitus as such is known to be an independent risk factor for CAD. On the other hand, CAD represents one of the major complications of a diabetic state where abnormalities in lipid metabolism are particularly prominent features. Framingham heart study shows that the impact of diabetes on CAD mortality is equivalent to that of a combination of any two of the other traditional risk factors such as hypertension and smoking. Multiple CVS risk factors are more common in diabetic patients than in non-diabetic patients. An analysis of all epidemiological studies on cardiovascular disease (CVD) and HCY in diabetes mellitus shows that there is no prospective work that specifically examined the relationship between levels of $\mathrm{t}-\mathrm{HCY}$ and the presence of CAD in the diabetes mellitus population. Thus additional studies are necessary to establish prospectively the presence of a multiplicative risk of CVD with elevated HCY levels in diabetes mellitus patients. ${ }^{[21]}$ Considering the controversy between hyperhomocysteinemia and CAD and the higher prevalence of CAD in patients with diabetes mellitus especially in Asian Indians, one of the objectives of the present investigation was to assess the HCY levels in patients with CAD.

It has been reported that hyperhomocysteinemia is caused by deficiency of folate, vitamin $B_{12}$, vitamin $B_{6}$ or impaired absorption of vitamin $B_{12}$ caused by gastric atrophy and many other factors. In a placebo-controlled study a combination of multiple agents including folic acid, vitamin $\mathrm{B}_{6}$ and vitamin $\mathrm{B}_{12}$ was very effective in reducing HCY levels in patients with moderate or intermediate hyperhomocysteinemia. ${ }^{[12]}$ However, folic acid is much strongly associated with HCY levels. ${ }^{[11]}$ In one study, 101 men with vascular disease were given supplementary doses of folic acid, vitamin $B_{6}$ and vitamin $B_{12}$. Ultrasound examination of their carotid arteries was done and a decrease in the amount of carotid plaque was found, especially in those whose HCY levels had been highest before treatment began. ${ }^{[2]}$ Thus the present investigation also aimed at determining the effect of folate treatment on elevated HCY levels.

\section{Materials and Methods}

The study was a controlled open clinical trial. It was randomized, non-blinded and based on parallel group design. The Institutional Review Committee of Sterling Hospital, Ahmedabad approved the study protocol. During the period from January to March 2002, 84 consecutive patients with or without diabetes mellitus who had undergone angiography at Sterling hospital, Ahmedabad and had angiographically documented CAD were enrolled for the study. Patients gave written informed consent before they were enrolled. Their age ranged from 30 to 70 years.

Patients above 70 years, or those with second or third degree AV block, renal or hepatic failure, recent cerebrovascular events, valve replacement surgery, severe dyslipidaemia (total cholesterol > $400 \mathrm{mg} / \mathrm{dl}$ ) or who underwent carotid bypass graft surgery were excluded from the study. The patients taking alcohol or vitamin B complex or who were non-vegetarian were also excluded.

Patients with CAD who met the eligibility criteria as mentioned above were divided into two groups as (1) diabetic, (2) non-diabetic. These patients were subdivided into: hypertensive and normotensive. In addition to these groups, we also had 40 volunteers who were treadmill test (TMT) negative (thus proved non-CAD) who were enrolled from the general population. Either TMT or angiography was done within $24 \mathrm{~h}$ before enrolling. These were also divided into four subgroups like those of the patients. 
All the patients were advised not to take fried, oily food after percutaneous transluminal coronary angioplasty (PTCA)/ coronary artery bypass graft surgery (CABG). They were asked to exercise daily with empty stomach. Patients were also asked to quit smoking after surgery. CAD patients underwent angiography so as to find out the severity of CAD before treatment. No angiography was done in volunteers however, they all were asked to undergo TMT.

First blood sample was collected before patient underwent folate treatment for the estimation of various biochemical parameters. The samples were analyzed for serum homocysteine levels by chemiluminiscence technique using Diagnostic reagent kit (Diagnostic Products Corporation, Los Angeles, USA); serum CRP by CRP latex test using Diagnostic reagent kit (Plasmatec Laboratory Products Limited, UK), serum glucose, total cholesterol, triglycerides, HDL, LDL and VLDL using Diagnostic reagent kits (Bayer India Ltd., Mumbai, India). Serum insulin was estimated by radioimmunoassay using the kits obtained from the Board of Radiation and Isotope Technology, Mumbai, India. Second blood sample was collected after three months of folate therapy $(5 \mathrm{mg} /$ day for 3 months) for the estimation of serum HCY.

\section{Statistical analysis}

The results were analyzed by applying Student's ' $t$ ' test, linear regression to find out the degree of correlation among the parameters studied and one-way analysis of variance (ANOVA). The value of probability less than $5 \%(P<0.05)$ was considered statistically significant.

\section{Results}

We included in our study 84 consecutive patients from the cardiology unit of Sterling Hospital. All of them had angiographically documented CAD and underwent PTCA or CABG. Thirty-five per cent of these patients were diabetic and $43 \%$ were hypertensive. We also included 40 control volunteers who were TMT negative and thus proved non-CAD. Among controls $52.5 \%$ were diabetic and 30\% were hypertensive (Table 1). It was interesting to note that $45 \%$ of the patients had family history of heart disease, which was higher than controls (30\%). Other characteristics like habit of smoking and tobacco chewing were not different between patients and controls. The HCY levels in CAD patients were found to be significantly $(P<0.0001)$ higher $(24.69 \pm 2.8 \mu \mathrm{mol} / \mathrm{L})$ than the con-

\section{Table 1}

Biographical characteristics of patients and controls

\begin{tabular}{lll}
\hline Characteristics & Control $\mathbf{n = 4 0}$ & Patients $\mathbf{n = 8 4}$ \\
\hline Age $(\mathrm{yr})$ & $46 \pm 1.5$ & $51 \pm 1.1$ \\
Female gender (\%) & 7.14 & 17.5 \\
Familial heart disease (\%) & 35 & 45.2 \\
Hypertension (\%) & 30 & 42.9 \\
Diabetes (\%) & 52.5 & 35.7 \\
Smoking (\%) & 5 & 21.4 \\
Tobacco (\%) & 10 & 15.5
\end{tabular}

Data presented are as mean \pm SEM, $\%$ of patients trol subjects $(17.85 \pm 1.4 \mu \mathrm{mol} / \mathrm{L})$.

Since diabetes and hypertension coexist we evaluated the HCY levels considering both diabetes and hypertension. The levels were found to be the least in the diabetic-hypertensive group of CAD patients (Figure 1), which were even less than their controls. Both CAD patients as well as control subjects with diabetes had lower HCY levels as compared to non-diabetics. All the non-diabetic patients had significantly higher HCY levels irrespective of whether they were hypertensive or not.

When the HCY levels were evaluated with respect to the presence of diabetes mellitus alone (Table 2) it was found that the levels were significantly higher in non-diabetic CAD patients as compared to all other groups $(\mathrm{F}=4.99, \mathrm{df}=3, P<0.05)$. Among diabetics, HCY levels were not significantly different between groups (i.e. patients or controls).

When hypertension was taken into consideration as an independent factor (Table 2), HCY levels were found to be significantly higher in non-hypertensive CAD patients as compared to their respective control.

The HCY levels were different in different age groups of patients. The levels were highest in the young age group (3040 years) of patients and as the age increased the levels de-

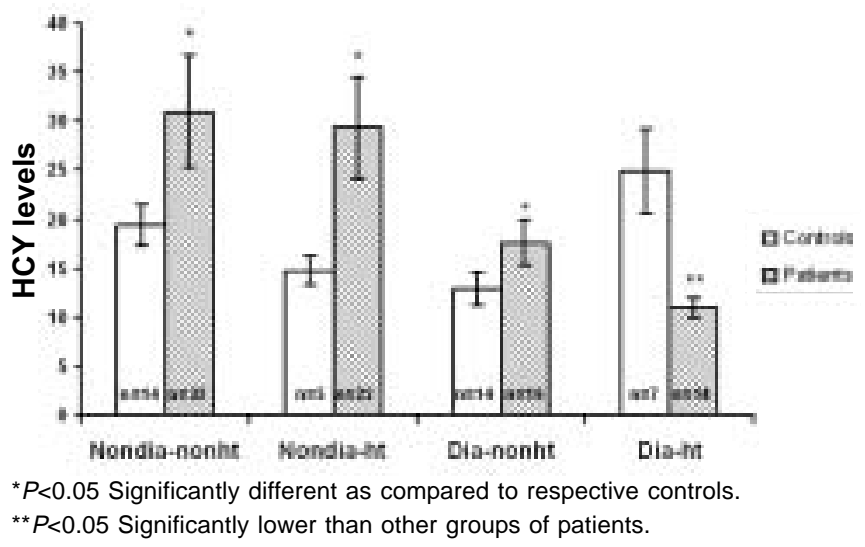

Figure 1: Homocysteine levels in various groups of patients and controls. Bars represent mean \pm SEM

Table 2

HCY levels in diabetic and hypertensive groups

\begin{tabular}{lcc}
\hline Group & No. of subjects & $\begin{array}{l}\text { HCY levels } \\
(\boldsymbol{\mu m o l} / \mathbf{L})\end{array}$ \\
\hline $\begin{array}{ccc}\text { Non-diabetic: } \\
\text { Control }\end{array}$ & 19 & $18.18 \pm 1.6$ \\
Patients & 54 & $30.33 \pm 3.9^{*}$ \\
Diabetic: & & \\
Control & 21 & $16.86 \pm 2.2$ \\
Patients & 30 & $14.53 \pm 1.4$ \\
Non-hypertensive: & & $16.12 \pm 1.4$ \\
Control & 28 & $27.3 \pm 4.0^{*}$ \\
Patients & 48 & $20.67 \pm 2.9$ \\
Hypertensive: & & $22.24 \pm 3.5$ \\
Control & 12 & \\
Patients & 36 & \\
\hline
\end{tabular}

*Significantly different as compared to respective control, $P<0.0001$ 
clined. This was not observed in controls (Table 3).

No correlation between the HCY levels and serum glucose, insulin, triglycerides, VLDL, LDL, HDL, cholesterol and CRP levels were found in CAD patients.

All the patients with HCY levels higher than $15 \mu \mathrm{mol} / \mathrm{L}$ were given folate $5 \mathrm{mg} /$ day, in addition to the drugs prescribed by doctors like aspirin, nitrates, beta-blockers, and diabetic patients were taking antidiabetic therapy additionally. After giving 3 months treatment with folate $(5 \mathrm{mg} /$ day), there was a decrease in HCY levels in patients with hyperhomocysteinemia [Normal range: 5-15 $\mu \mathrm{mol} / \mathrm{L}$, moderate risk: $15-30 \mu \mathrm{mol} / \mathrm{L}$, intermediate risk: 30-100 $\mu \mathrm{mol} / \mathrm{L}$, severe: $>100 \mu \mathrm{mol} / \mathrm{L}$ (Kang et al., 1992)] (Table 4).

\section{Discussion}

In the present investigation HCY levels in the CAD patients were found to be higher $(24.69 \pm 2.8 \mu \mathrm{mol} / \mathrm{L})$ than those in the control subjects $(17.85 \pm 1.4 \mu \mathrm{mol} / \mathrm{L})$. This is consistent with the findings from several epidemiological and experimental studies. ${ }^{\mid 9,13]}$

Plasma HCY levels increase with advancing age in both sexes. ${ }^{[23]}$ In the present study we found that the levels of HCY were higher in the younger age group of patients. The levels were significantly higher as compared to those in controls. But as the age increased the levels were found to decrease.

\section{Table 3}

\section{HCY levels in different age groups}

\begin{tabular}{ccc}
\hline Age group & No. of subjects & HCY levels $(\boldsymbol{\mu m o l} / \mathbf{L})$ \\
\hline 30-40 years: & 12 & $17.3 \pm 2.2$ \\
Control & 14 & $49.0 \pm 7.8^{*}$ \\
Patients & & \\
41-50 years: & 13 & $15.8 \pm 9.0$ \\
Control & 27 & $27.4 \pm 6.1$ \\
Patients & & \\
51-60 years: & 13 & $18.5 \pm 3.2$ \\
Control & 26 & $15.1 \pm 1.5$ \\
Patients & & \\
61-70 years: & 2 & $22.9 \pm 3.6$ \\
Control & 17 & $14.4 \pm 1.4$ \\
Patients & & \\
\hline
\end{tabular}

*Significantly higher than other groups, $F=9.064, d f=3, P<0.0001$

\section{Table 4}

Effect of folate treatment on hyperhomocysteinemia

\begin{tabular}{|c|c|c|}
\hline \multirow[t]{2}{*}{ Group } & \multicolumn{2}{|c|}{ HCY levels $(\mu \mathrm{mol} / \mathrm{L})$} \\
\hline & Initial & After treatment \\
\hline $\begin{array}{l}\text { Mild hyperhomocysteinemia } \\
\text { (HCY levels } 16-30 \mu \mathrm{mol} / \mathrm{L})\end{array}$ & $\begin{array}{l}20.77 \pm 0.8 \\
(n=26)\end{array}$ & $\begin{array}{l}12.61 \pm 0.77^{*} \\
(n=21)\end{array}$ \\
\hline $\begin{array}{l}\text { Moderate hyperhomocystinemia } \\
(\mathrm{HCY} \text { levels } 31-100 \mu \mathrm{mol} / \mathrm{L})\end{array}$ & $\begin{array}{l}48.66 \pm 4.66 \\
(n=16)\end{array}$ & $\begin{array}{l}28.29 \pm 2.62^{*} \\
(n=14)\end{array}$ \\
\hline $\begin{array}{l}\text { Severe hyperhomocysteinemia } \\
(\mathrm{HCY} \text { levels }>100 \mu \mathrm{mol} / \mathrm{L})\end{array}$ & $\begin{array}{l}137.5 \pm 32.59 \\
(n=4)\end{array}$ & $\begin{array}{l}105 \pm 15.04 \\
(n=4)\end{array}$ \\
\hline
\end{tabular}

*Significant decrease in $\mathrm{HCY}$ levels as compared to the initial values, $P<0.05$
Thus it can be assumed that hyperhomocysteinemia is a major risk factor in young age CAD patients.

Various other risk factors for CAD are hypercholesterolemia, hypertriglyceridaemia, hyperinsulinemia, C-Reactive Protein, and abnormal glucose levels. ${ }^{[24]}$ It has been known that the lipid status is only weakly associated with HCY levels. ${ }^{[23]}$ In the present study we found that none of the factors (serum glucose, insulin, VLDL, LDL, HDL, CRP, TG and cholesterol) showed any correlation with the serum HCY levels.

Diabetes mellitus is a major risk factor for CAD. ${ }^{[25]}$ We evaluated our patients according to the presence or absence of diabetes. Prospective studies ${ }^{[26,27 \mid}$ suggested a stronger causal relationship between hyperhomocysteinemia and all cause mortality in diabetic patients than in non-diabetic ones. But the causal relationship was less clear for cardiovascular morbidity and mortality. The mean plasma t-HCY level is usually low or normal in diabetes mellitus patients, except when nephropathy is present. ${ }^{\mid 211}$ In our investigation we found that the HCY levels in diabetic CAD patients were low $(14.53 \pm 1.4 \mu \mathrm{mol} /$ L) as compared to non-diabetic CAD patients ( $30.33 \pm 3.9 \mu \mathrm{mol} /$ L). These findings indicate that even though diabetes and hyperhomocysteinemia are strong risk factors of $\mathrm{CAD}$, there is no synergistic relationship between the two, rather, diabetes decreases HCY levels. Decrease in the HCY level in diabetics may be due to glomerular hyper-filtration in diabetes. ${ }^{[26]}$ The precise mechanism is yet to be evaluated. When hypertension was taken into consideration, HCY levels were not significantly different in normotensive as compared to hypertensive CAD patients. However, when we segregated our patients based on the coexistence of diabetes and hypertension we found that the levels were less in the diabetic-hypertensive group of patients, which were even less than their controls. Various agents like vitamin $\mathrm{B}_{6}$, vitamin $\mathrm{B}_{12}$, trimethylglycine, folate may be used to bring down HCY levels. There is a clear inverse correlation of t-HCY with folate and vitamin $B_{12}$ stores ${ }^{[28]} \mathrm{A}$ similar but lesser effect is seen with vitamin $\mathrm{B}_{12}{ }^{{ }^{128]}}$ The degree of HCY elevation depends on the degree of vitamin deficiency. But folic acid supplementation is the major therapeutic intervention for the treatment of elevated HCY levels. Our findings are also in accordance with the earlier reports. ${ }^{[29]}$ When we analyzed the HCY levels in the hyperhomocysteinemic patients after 3-month treatment with folic acid, we found significant reduction in the HCY levels in patients with mild and moderate hyperhomocysteinemia but the levels did not decrease significantly in severe hyperhomocsyteinemic patients.

Reduction in HCY may improve endothelial function. Woo et al $^{[30]}$ showed improved endothelium-dependent vasodilation after folic acid supplementation in hyperhomocysteinemic subjects. Whether this improvement is due to the HCY-lowering effect of folic acid or is due to other properties of folic acid is unclear. More prospective studies are thus required to evaluate the exact mechanism of action.

In conclusion, our data suggest that (a) hyperhomocysteinemia appears to be an independent risk factor for CAD in younger patients. (b) Patients with diabetes mellitus show low levels of t-HCY irrespective of the presence of cardiac disease that might interfere with the correlation of hyperhomocysteinemia and CAD. (c) Folate decreases the tHCY levels in homocysteinemia and thus can be used in the 
treatment of homocysteinemia. However, further studies with a large sample size may be required to support the above conclusions.

\section{References}

1. Enas EA, Mehta JL. Malignant Coronary Artery Disease in young Asian Indians. Thoughts on Pathogenesis, prevention and therapy. N Engl J Med 1995:333:1301-7.

2. Sethi KK. Homocysteine: An Easily Reversible Risk Factor for A-Atherosclerosis. Quartely J Cardiol 2002;1:7A-11A.

3. Bulatao RA, Stephens PW. Global estimates and projections of mortality by cause. Washington, DC: Population, Health and Nutrition Department World bank, Preworking paper; 1992.

4. Krishnaswami S, Prasad NK, Jose VJ. A study of lipid levels in Indian patients with coronary artery disease. Int J Cardiol 1989;24:337-45.

5. Hughes LO. Insulin, Indian origin and ischemic heart disease. Int J Cardiol 1990;26:1-4.

6. Vardan S, Mukherjee S, Vardan S, Sinha AKN. Special features of coronary heart disease in people of Indian subcontinent. Indian Heart J 1995;47:399-407.

7. Mehta J, Mehta P, Lowsen D, Salden T. Plasma tissue plasminogen activator inhibitor levels in coronary artery disease: Correlation with age and serum triglyceride concentrations. J Am Coll Cardiol 1987;9:263-8.

8. Kannel WB. Range of serum cholesterol values in the population developing coronary artery disease. Am J Cardiol 1995;76:69-77.

9. Clarke R, Daly L, Robinson K, Naughten E, Cahalane S, Fowler B, et al. Hyperhomocysteinemia: An independent risk factor for vascular disease. $\mathrm{N}$ Engl J Med 1991;324:1149-55.

10. Perry IJ, Refsum H, Moris RW, Ebrahim SB, Ueland PM, Shaper AG. Prospective study of serum total homocysteine concentration and risk of stroke in middle-aged British men. Lancet 1995;346:1395-8.

11. Boushey CJ, Beresford SAA, Omenn GS, Motusky AG. A quantitative assessment of plasma homocysteine as a risk factor for vascular disease: Probable benefits of increasing folic acid intakes. J Am Med Assoc 1995;247:1049-57.

12. Ubbink JB, Becker PJ, Vermaak WJ, Delport R. Results of B-vitamin supplementation study used in a prediction model to define a reference range for plasma homocysteine. Clin Chem 1995;41:1033-7.

13. McCully KS. Vascular pathology of homocysteinemia, implications for pathogenesis of artheriosclerosis. Am J Pathol 1969;56:111-28.

14. Tsai JC, Perrella MA, Yoshizumi M, Hsieh CM, Haber E, Schlegel R, et al. Promotion of vascular smooth muscle cell growth by homocysteine: A link to atherosclerosis. Proc Natl Acad Sci USA 1994;91:6369-73.

15. Woo KS, Chook P, Lolin YI, Cheung AS, Chan LT, Sun YY, et al. Hyperhomocyst(e)inemia is a risk factor for arterial endothelial dysfunction in humans. Circulation 1997:96:2542-4.

16. Petri M, Roubenoff R, Dallal GE, Nadeau MR, Selhub J, Rosenberg IH. Plasma homocysteine as a risk factor for atherothrombotic events in systemic lupus erythematosus. Lancet 1996;348:1120-4.

17. Anand SS, Yusuf S, Vuksan V, Devanesen S, Teo KK, Montague PA, et al. Differences in risk factors, atherosclerosis, and cardiovascular disease between ethnic groups in Canada: The Study of Health Assessment and Risk in Ethnic groups (SHARE). Lancet 2000;356:279-84.

18. Chambers JC, Obeid OA, Refsum H, Ueland P, Hackett D, Hooper J, et al. Plasma homocysteine concentrations and risk of coronary heart disease in UK Indian Asian and European men. Lancet 2000;355:523-7.

19. Deepa R, Velmurugan K, Saravanan G, Karkuzhali K, Dwarakanath V, Mohan $\mathrm{V}$. Absence of association between serum homocysteine levels and coronary artery disease in South Indian males. Indian Heart J 2001;53:44-7.

20. Gupta MD, Girish MP, Nigam A, Roy TNS, Sharma A, Tyagi S, et al. Hyperhomocysteinemia as a Risk Factor for Angiographic Coronary Artery Disease: Observations from a North Indian Community. Indian Heart J 2003;55:229.

21. Audelin MC, Genest J. Homocysteine and cardiovascular disease in diabetes mellitus. Atherosclerosis 2001;159:497-511.

22. Genest J Jr, Audelin MC, Lonn E. Homocysteine: To screen and treat or to wait and see? Can Med Assoc J 2001;163:37-8.

23. Nygard $\mathrm{O}$, Refsum $\mathrm{H}$, Ueland PM, Vollest SE. Major lifestyle determinants of plasma total homocysteine distribution: The Hordaland Homocysteine Study. Am J Clin Nutr 1998;67:263-70.

24. Mendall MA, Patel P, Ballam L, Strachan D, Northfield TC. C-reactive Protein and its relation to cardiovascular risk factor: A population based cross sectional study. Br Med J 1996;312:1061-5.

25. Grundy SM, Benjamin IJ, Burke GL, Chait A, Eckel RH, Howard BV, et al. Diabetes and cardiovascular disease: A statement for healthcare professionals from the American Heart Association. Circulation 1999;100:1134-46.

26. Araki A, Sako Y, Ito H. Plasma homocysteine concentrations in Japanese patients with non-insulin-dependent diabetes mellitus. Effect of parental methylcobalamin treatment. Atherosclerosis 1993;103:149-57.

27. Chambers JC, Obeid O, McGregor A, Powell-Tuck J, Boustead L, Kooner JS. The relationship between hyperhomocysteinemia and endothelial dysfunction is concentration-dependent and present even at physiological levels. Circulation 1998;98:I-192.

28. Langman LJ, Cole EC. Homocysteine: Cholesterol of the 90s? Clin Chim Acta 1999;286:63-80.

29. Refsum H, Ueland PM, Nygard O, Vollset SE. Homocysteine and cardiovascular disease. Annu Rev Med 1998;49:31-62.

30. Woo K, Chook P, Lonin Y, Sanderson JE, Metreweli C, Celermajer OS. Folic acid improves arterial endothelial function in adults with hyperhomocysteinemia. J Am Coll Cardiol 1999;34: 2002-6.

\section{Join "IndPharm" \\ IJP uses "IndPharm" to broadcast announcements. Want to join? Please E-mail: adithan@vsnl.com}

